\title{
Torturing the helpless: A review of PCOS induced infertility from a gender perspective
}

\begin{abstract}
This paper reviewed the abuse of infertile women suffering from Polycystic Ovary Syndrome (PCOS) from a gender perspective. Infertility is a prevalent, presenting feature of PCOS with $75 \%$ of women experiencing infertility due to anovulation, making PCOS the most common cause of anovulatory infertility. Increased awareness of PCOS, its causes, and its symptoms may help the process of early diagnosis, appropriate care and mitigation of violence arising from infertility hence, this study. In many countries' infertility among married couples especially for women is a sentence to stigmatization, loss of social status, marital conflicts and violence. All this stems from prevailing socio-cultural norms and gender inequalities inimical to women. PCOS is a syndrome without much public awareness and PCOS patients often do not seek care. Where they seek care, they are often not immediately diagnosed with PCOS. Due to some prevailing cultural norms and general lack of awareness they are often tortured and abused. Outcomes from this study shows that there is need to intensify public awareness on the various factors contributing to infertility such as PCOS which has been identified as a major contributing factor. Also, harmful socio-cultural norms and practices that encourages gender inequalities and violence against infertile women should be eradicated with strong policies put in place and perpetrators severely punished. Early diagnosis and a multidisciplinary approach to the treatment of PCOS is also crucial. Finally, proactiveness by implementing working strategies that will help improve treatment and mitigate violence against women suffering from PCOS should be embrace by all.
\end{abstract}

KEYWORDS: PCOS, gender, gender based violence, infertility, gender inequality, torture

\section{Introduction}

Infertility is a major factor that underlie domestic violence against women (Poornowrooz et al., 2019). Due to gender-based inequalities arising from socially constructed norms and values, women are often discriminated against and bear the burnt for infertility (Patel et al., 2018). Generally, infertility causes a variety of psychosocial and cultural problems for women as they are often humiliated and violently abused as a result (Akyuz et. al., 2013; Ozgoli et al., 2016; Tazuh \& Tosam, 2016). In male-dominated societies, such as is found in sub-Saharan Africa, the woman is consistently 
singled out and blamed for a couple's infertility, she is often severely punished socially and economically and made highly vulnerable to violence (Ibisomi \& Mudege 2014; Tazuh \& Tosam, 2016). Fortunately, most cases of infertility in which women are humiliated and abused for, is treatable most times when the cause is identified early and adequately attended to. Unfortunately, a lot of people are unaware of this and still go ahead and violently abuse women for these.

Polycystic Ovary Syndrome (PCOS) is a major cause of infertility and unintentional childlessness among premenopausal women (Bellver et al., 2018). PCOS is generally defined as hyperandrogenism associated with chronic anovulation in women without other underlying disease (Brady et al., 2009). At any age, PCOS can be devastating to women, especially during the reproductive years as it is a leading and major cause of female infertility (Costello et al., 2019). Infertility is a prevalent presenting feature of PCOS with $75 \%$ of these women experiencing infertility due to anovulation, making PCOS the most common cause of anovulatory infertility (Brady et al., 2009; Costello et al., 2019). As a matter of fact, the prevalence of infertility in women with PCOS is between 70 and $80 \%$ but many women are often not immediately diagnosed with PCOS, which makes them to suffer long for infertility which can be treated to vindicate them (Melo, Ferriani \& Navarro, 2015). In many countries, it represents the leading cause of female infertility (Matta, 2017). PCOS also represent a major stress factor in the life of the female gender (Tan et al., 2008; Damone et al., 2019) and women suffering from PCOS reportedly suffer from marked reductions in quality-of-life, violent abuse, emotional distress and increased incidence of depression (Damone et al., 2019). Several studies (Aduloju et al., 2015) have shown that infertile women (PCOS inclusive) are highly susceptible to some underlying factors such as depression, anxiety and stressful events that predispose them to domestic violence. Thus, given its prevalence PCOS represents a major risk factor for violence against women.

Although PCOS is a major public health problem (Soni, 2017; Kirthika et al., 2019), referred to as one of the most common endocrine disorders in premenopausal women, yet it is a condition the public is largely unaware of and health care providers do not seem to fully understand (Azziz, 2016). Similarly, a lack of awareness about the disease and associated infertility predispose a lot of women to violent abuse, torture and unwarranted suffering, especially in sub-Saharan Africa where children are seen as status symbol. Due to lack of public awareness majority of PCOS patients often do not seek medical care (Sanchez, 2014). This pose a challenge for the sufferers as they 
are prone to violence, torture and various psychological disorders such as sadness, anger, depression and humiliation. Due to ignorance and lack of adequate information some people do erroneously believe that women with PCOS can never conceive (Sharma \& Mishra, 2018). However, doctors think differently towards this statement, because, in some cases, women with PCOS do get pregnant even without any medical assistance and in some cases, just a little medical assistance has proven to be helpful (Sharma \& Mishra, 2018).

While men and women are assumed to have equal probability of being infertile, in many societies of Africa the problem of infertility is seen mainly as a woman's problem (Bayouh, 2011). In such societies, women suffer severe stigmatization and violence since they are presumed to have failed to conceive. A woman's infertility may lead to violence, rejection by her partner, social exclusion, and loss of access to resources (Agbontaen-Eghafona \& Ofovwe, 2009). Women without children are considered not to be fully adult and are presumed to be of minimal economic and social value in terms of household wealth or lineage continuity. All these are due to prevailing socio-cultural gender constructs. Gender used in the context of this study refers to social cultural norms or traditions that shape our behaviors, preferences and knowledge. Deconstructing prevailing gender norms through public awareness and education is crucial in mitigating violence against women due to PCOS induced infertility.

Given the high prevalence of PCOS (Matta, 2017), and the fact that infertility is one of the main implications of the diagnosis (Costello et al., 2019), examining the associated socio-cultural consequences with the objective of increasing awareness and proffering solutions from a gender perspective certainly deserves further study. This paper is a review of PCOS induced infertility among women from a gender perspective. This is with an aim to increase awareness on PCOS induced infertility thereby mitigating gender-based violence against women as a result of PCOS induced infertility. In addition, the outcomes from this study are relevant to policy makers in sub-Saharan Africa and other developing countries who are interested in addressing gender-based violence, human rights issues and public health consequences associated with PCOS.

\section{Prevalence of violence associated with infertility}

Gender-based violence, though under reported, affects the lives of millions of women and young girls worldwide notwithstanding their socioec- 
onomic or educational backgrounds (Ozturk et al., 2017). Violence against women, especially against the more vulnerable infertile group must be handled as a public health problem, which negatively affects the reproductive and sexual health rights of women (Eka, Swende \& Hembah-Hilekaan, 2019). The prevalence of violence ranges between $15 \%-71 \%$ worldwide (WHO, 2017). Global estimates published by WHO showed that about one third (35\%) of women worldwide had experienced either physical and/or sexual violence in their lifetime (WHO, 2017). Violence affects women of all ages, races, and backgrounds (Ozturk et al., 2017).

\section{Implications of infertility in sub-Saharan Africa}

In sub-Saharan Africa (SSA), approximately $1.9 \%$ of the population are confronted with primary infertility which occurs when a woman is unable to bear a child, either due to the inability to become pregnant or the inability to carry a pregnancy to a live birth (Dierickx et al., 2018). Also, there continues to be a high rate of secondary infertility which occurs when a woman is unable to bear a child, either due to the inability to become pregnant or carry a pregnancy to a live birth following either a previous pregnancy or a previous ability to carry a pregnancy to a live birth (Dierickx et al., 2018).

Existing research on infertility in SSA suggests that it has important implications for mental health, economic and social well-being, marital quality and stability (Fledderjohann, 2012). However, it is vital to note that many of these findings are likely to vary across the different cultural and social settings. Reproduction is a natural yearning and an important human need and generally considered to be the most important element for enduring marital relationship (Sami \& Ali, 2012) especially in some cultural settings. The desire to have children is strong and compelling, so infertility can be devastating for couples who are unable to conceive. A woman who gives birth to a healthy child is highly valued in her society. Consequently, infertility is not only a gynaecological illness but also a socio-cultural health challenge (Harzif, Santawi \& Wijaya, 2019). Infertility also poses as a social stigma (Harzif, Santawi \& Wijaya, 2019). Several studies have shown the negative effects of infertility from the social point of view, this negative social problem affects the lives of millions of women worldwide (Harzif, Santawi \& Wijaya, 2019). Various anthropological and sociological research have also shown that due to prevailing cultural and 
gender norms towards childbearing and infertility, women in SSA countries are often more negatively affected and prone to violence (Dierickx et al., 2018). In SSA societies with high levels of gender inequality, regardless of diagnosis, women are blamed for failing to bear children (Dierickx et al., 2018). They do suffer from stigma, social isolation and ridicule within their communities as a result of infertility. Fertility problems are also a potential source of marital tension and violence between partners (Dierickx et al., 2018). When a marriage remains childless, the woman is more prone to verbally or even physical violence. They are prone to exhibit feelings of anxiety, lack of self-esteem and a general sense of powerlessness. This is mostly common in patriarchal societies, where the role of women is determined according to their capacity to give birth, thus making infertility a major threat to marital life stability (Inhorn \& Patrizio, 2015). This has serious consequences for a woman's total well-being as a result of the fact that motherhood in patriarchal societies, is a confirmation of a woman's feminine as well as maternal identities.

Furthermore, in such patriarchal societies infertile women are highly vulnerable to various forms of violence especially domestic violence. Where parenthood is considered as a master status by the individual or by the society, inevitable distress as a result of infertility is the consequence (Hess, Ross \& Gililland, 2018). Women for whom motherhood is defined as a major life identity experience, do, significantly have higher levels of psychological distress when they remain childless (Hess, Ross \& Gililland, 2018). In most SSA countries, social discrimination and domestic violence against infertile women by their spouses and spouses' relatives are widespread (Dierickx et al., 2018).

\section{Prevalence of PCOS induced infertility}

The true prevalence of PCOS is generally unknown (Cutler, 2019) as nationwide data is scanty especially in SSA. Reports vary depending on the diagnostic criteria used and the population studied. Worldwide estimates report between 6 to 18\% of women have PCOS (Cutler, 2019). While PCOS affects women worldwide, some evidence suggests higher rates in specific ethnic groups (Wolf et al., 2018).

As noted earlier, prevalence depends on the diagnostic criteria being used. For example, the 2003 Rotterdam criteria are the broadest and therefore yields the highest rates of PCOS while the $1990 \mathrm{NIH}$ criteria are the 
strictest (Cutler, 2019). According to Cutler 2019, a systematic review and meta-analysis comprising of 13 studies where ethnicity was categorized as follows: African American, European White, Chinese and Middle Eastern; was carried out. In this meta-analysis, prevalence using the Rotterdam criteria was $5.6 \%$ for Chinese women and $16.0 \%$ for Middle Eastern women (Cutler, 2019). Using the NIH criteria, results for prevalence rates was 5.5\% for European White women, 7.4\% for African American women and $6.1 \%$ for Middle Eastern women (Cutler, 2019). Meanwhile, estimated prevalence was $12.6 \%$ for Middle Eastern women using the 2006 Androgen excess (AE-PCOSS) criteria (Cutler, 2019). These prevalence rates point to African American women having the highest prevalence of PCOS while Chinese women having the lowest (Cutler, 2019).

\section{Infertility and PCOS: interrelations of gender roles and sociocultural expectations}

Infertility is socially constructed in many SSA cultures, that is, men and women are viewed and perceived to become parents and women are specially socialized to become mothers in society (Chimbatata \& Malimba, 2016). This is an unambiguous contrast to other societies in the west and the affluent world where individuals or couples could choose not to have a child or just to have them by adoption. Infertility has psychological and social aspects as well as being a medical problem (Kazandi et al., 2011). It is a problem in social contexts because in most societies of the world, womanhood and manhood are generally associated with childbearing (Tabong \& Adongo, 2013). PCOS as a condition has an effect on virtually every aspects of a woman's life. Although the health aspect is being extensively worked on since the past few decades, little has been done to address the social cultural aspects of the condition especially from a gender lens. In many SSA countries where the socio-cultural environment is patriarchal in their approach, there are serious social-cultural consequences in the life of a woman with PCOS induced infertility.

In most SSA countries in general, women enjoy less status than men. Sub-Saharan African culture is majorly pronatalist and choosing voluntary childlessness is rare (Reed, 2010). Most men and women place a great deal of importance on having children especially in SSA where the high infant and child mortality rate motivates couples to have as many children as possible (Reed, 2010). Across SSA infertile women are subjected to public 
derogation and violence (Reed, 2010). Infertility due to PCOS make women more vulnerable to violence due to inherent social cultural norms and gender inequalities that predisposes them to such. Unfortunately, majority of women affected by PCOS are unaware of their situation and the level of societal awareness is still quite low. Aside the violence, the societal perception of infertile women is negative especially in SSA. They need help and societal support thus, inherent negative perceptions and violence towards them is akin to torturing the helpless.

\section{The way forward}

\section{Increased awareness}

Awareness is the first step in protecting women from violence due to PCOS induced infertility. Little is known about the specific fertility concerns and information needs of women with PCOS or their preferences for how and when to receive information about the effect of their condition and its treatment on fertility and childbearing (Holton, Hammarberg \& Johnson, 2018). Women with PCOS want to be better informed about the impact of their condition on their reproductive capacity but find it challenging to access reliable, relevant and timely information (Holton, Hammarberg \& Johnson, 2018). The primary cause of PCOS is still unknown but awareness and lifestyle modifications are known to be an effective therapy route to ameliorate the symptoms of this syndrome (Cutler, 2019; Rao, Broughton \& Lemieux, 2020).

Furthermore, the lack of public awareness results in many not seeking healthcare or going undiagnosed (Rao, Broughton \& Lemieux, 2020). In many SSA countries, the lack of public awareness of this disease has contributed to its low clinical diagnosis rate as most patients do not seek adequate and proper health care (Cutler, 2019). Increase PCOS awareness (both among physicians and patients) should result in more women seeking medical evaluation specifically because PCOS is suspected (Rao, Broughton \& Lemieux, 2020). According to Dr. Ilana Ressler, "PCOS goes undiagnosed in $50 \%-70 \%$ of women who have the condition" (Levinson, 2019) and PCOS is one of the most underserved women's health issues today due to lack of sufficient public awareness (Cutler, 2019). Thus, efforts to heighten the profile of PCOS among the general public remain critical, as "minimal awareness" or "no awareness" of PCOS is prevalent. 


\section{More studies on the socio-cultural impact of PCOS}

Only a very few studies have highlighted the socio-cultural impact of PCOS induced infertility especially pertaining to violence against women in sub-Saharan Africa. This is particularly alarming given the need to improve women's health in the region and the great need to address health disparities globally (Mariani et al., 2017). The negative impact that PCOS has on fertility will be particularly harmful for African women in low socioeconomic settings. Many are affected by psychosocial effects such as intimate partner violence, anxiety and depression, stress, and divorce (Zangeneh et al., 2012). There is need for more efforts to create more public awareness on PCOS especially in sub-Saharan Africa as this will also motivate more studies to be carried out in this direction.

\section{Multifaceted approach to treatment}

Solutions for improving care for women with PCOS need to be multifaceted (Teede et al., 2018; Wright, Dawson \& Corbett, 2020). A multidisciplinary and multilevel approach for managing PCOS is necessary. Women with PCOS should receive empathic, supportive care that speaks to all aspects of their condition. This should be incorporated as a policy while treating women with PCOS induced infertility. Women suffering from PCOS should be given appropriate and wholistic care, rather than being consistently tortured and humiliated.

\section{Policy and societal reorientation}

Infertility is wrongly seen as a woman's issue in many SSA settings with its disruptive power towards women (Chimbatata \& Malimba, 2016). If the fight against PCOS induced infertility is to be won, then all concerned must take responsibility and act appropriately. This requires more vigorous public awareness and educational campaigns especially at the grassroot level. The society's over valuing of children and projecting those who are childless as useless in society needs to be critically reexamined (Chimbatata \& Malimba, 2016). It is such traditional perception that puts more pressure on concerned infertile individuals and encourage violence against woman. If care for women with PCOS is to improve, there is need for policy change that would break the traditional norm of viewing infertility as solely a woman's issue.

The perception of infertility as something that would deny individuals communal rights also requires to be changed (Chimbatata \& Malimba, 
2016). The society should give equal respect to individuals regardless to whether they have children or not (Chimbatata \& Malimba, 2016). This will encourage positive acceptance of infertility as a reproductive health problem and not a punishment (Chimbatata \& Malimba, 2016). This will go a long way in reducing violence against women due to PCOS induced infertility.

\section{Relieving the economic burden}

The economic burden of PCOS is significantly large especially for women in developing countries where the poverty rate is high. Thus, early diagnosis and intervention is crucial to reduce the financial burden and ensure good health. This is important as it will ultimately reduce incidences of violence against women and ensure increased economic productivity.

\section{Conclusion}

The current state of awareness and knowledge regarding PCOS induced infertility is quite low especially in SSA. Some existing societal norms and gender inequalities increases the vulnerability of women suffering from fertility problems to violence. Outcomes from this review shows that there is a great need for more advocacy and increased awareness on PCOS induced infertility. Furthermore, harmful socio-cultural practices and gender inequalities that make infertile women more vulnerable to violence should be eradicated through advocacy and appropriate legislations. Also, early diagnosis and a multidisciplinary approach to the treatment of PCOS is crucial. Finally, government, traditional institutions and the society in general need to be more proactive by implementing evidence-based strategies that will help improve treatment and mitigate violence against women suffering from PCOS.

\section{REFERENCES}

ADULOJU, P. O. et al. (2015) Prevalence and predictors of intimate partner violence among women attending infertility clinic in south-western Nigeria. European Journal of Obstetrics \& Gynecology and Reproductive Biology. 188. Pp. 66-69.

AGBONTAEN-EGHAFONA, K. A. \& OFOVWE, C. E. (2009) Infertility in Nigeria: A risk factor for gender-based violence. Gender and Behaviour. 7 (2). Pp. 2326-2344. 
AKYUZ, A. et al. (2013) Studying the effect of infertility on marital violence in Turkish women. International Journal of Fertility \& Sterility. 6 (4). Pp. 286.

AZZIZ, R. (2016) Introduction: Determinants of polycystic ovary syndrome. Fertility and Sterility. 106 (1). Pp. 4-5.

BAYOUH, F. A. (2011) Socio-cultural perceptions of infertility and their implications: a study of women experiencing childlessness in South Gondar, Ethiopia (Master's thesis, The University of Bergen).

BELLVER, J. et al. (2018) Polycystic ovary syndrome throughout a woman's life. Journal of Assisted Reproduction And Genetics. 35 (1). Pp. 25-39.

BRADY, C., MOUSA, S. S. \& MOUSA, S. A. (2009) Polycystic ovary syndrome and its impact on women's quality of life: More than just an endocrine disorder. Drug, Healthcare and Patient Safety. Vol. 1. Pp. 9-15.

CHIMBATATA, N. B. \& MALIMBA, C. (2016) Infertility in sub-Saharan Africa: a Woman's issue for how long? A qualitative review of literature. Open Journal of Social Sciences. 4 (8). Pp. 96-102.

COSTELLO, M. et al. (2019) A review of first line infertility treatments and supporting evidence in women with polycystic ovary syndrome. Medical Sciences. 7 (9). Pp. 95.

CUTLER, D. (2019) The impact of lifestyle on the reproductive, metabolic, and psychological well-being of women with polycystic ovary syndrome (PCOS) (Doctoral dissertation, University of British Columbia).

DAMONE, A. L. et al. (2019) Depression, anxiety and perceived stress in women with and without PCOS: a community-based study. Psychological Medicine. 49 (9). Pp. 1510-1520.

DIERICKX, S. et al. (2018) 'I am always crying on the inside': a qualitative study on the implications of infertility on women's lives in urban Gambia. Reproductive Health. 15 (1). P. 151.

EKA, P. O., SWENDE, T. Z. \& HEMBAH-HILEKAAN, S. K. (2019) Domestic Violence among Infertile Women in Makurdi, North-Central Nigeria. Gynecol Reprod Health. 3 (5). Pp. 1-4.

FLEDDERJOHANN, J. J. (2012) 'Zero is not good for me': implications of infertility in Ghana. Human Reproduction. 27 (5). Pp. 1383-1390.

HARZIF, A. K., SANTAWI, V. P. A. \& WIJAYA, S. (2019) Discrepancy in perception of infertility and attitude towards treatment options: Indonesian urban and rural area. Reproductive Health. 16 (1). P. 126.

HESS, R. F., ROSS, R. \& GILILLAND JR, J. L. (2018) Infertility, psychological distress, and coping strategies among women in Mali, West Africa: a mixed-methods study. African Journal of Reproductive Health. 22 (1). Pp. 60-72.

HOLTON, S., HAMMARBERG, K. \& JOHNSON, L. (2018) Fertility concerns and related information needs and preferences of women with PCOS. Human Reproduction Open. (4).

IBISOMI, L. \& MUDEGE, N. N. (2014) Childlessness in Nigeria: perceptions and acceptability. Culture, Health \& Sexuality. 16 (1). Pp. 61-75.

INHORN, M. C. \& PATRIZIO, P. (2015) Infertility around the globe: new thinking on gender, reproductive technologies and global movements in the $21^{\text {st }}$ century. Human Reproduction Update. 21 (4). Pp. 411-426. 
KAZANDI, M. et al. (2011) The status of depression and anxiety in infertile Turkish couples. Iranian Journal of Reproductive Medicine. 9 (2). P. 99.

KIRTHIKA, S. V. et al. (2019) Polycystic ovarian syndrome-interventions for the emerging public health challenge: A scoping review. Drug Invention Today. 12 (3). Pp. 1-4.

LEVINSON, A. (2019) Raising Awareness and Requesting Funding for PCOS-The \#1 Infertility Diagnosis. [Online] Available from: https://www.rmact.com/fertility-blog/raising-awareness-and-requesting-funding-pcos [Accessed: 9 September 2020].

MARIANI, G. et al. (2017) Improving women's health in low-income and middle-income countries. Part I: challenges and priorities. Nuclear Medicine Communications. 38 (12). P. 1019.

MATTA, R. A. (2017) Apelin-36 and Copeptin Levels in Polycystic Ovary Syndrome. Journal of Infectious Diseases. 3 (1). P. 3.

MELO, A. S., FERRIANI, R. A. \& NAVARRO, P. A. (2015) Treatment of infertility in women with polycystic ovary syndrome: approach to clinical practice. Clinics. 70 (11). Pp. 765-769.

OZGOLI, G. et al. (2016) Evaluation of the prevalence and contributing factors of psychological intimate partner violence in infertile women. Journal of Midwifery and Reproductive Health. 4 (2). Pp. 571-581.

OZTURK, R. et al. (2017) Another face of violence against women: Infertility. Pakistan Journal of Medical Sciences. 33 (4). P. 909.

PATEL, A. et al. (2018) Sociocultural determinants of infertility stress in patients undergoing fertility treatments. Journal of Human Reproductive Sciences. 11 (2). Pp. 172.

POORNOWROOZ, N. et al. (2019) The Comparison of Violence and Sexual Function between Fertile and Infertile Women: A Study from Iran. Journal of Clinical \& Diagnostic Research. 13 (1).

RAO, M., BROUGHTON, K. S. \& LEMIEUX, M. J. (2020) Cross-sectional Study on the Knowledge and Prevalence of PCOS at a Multiethnic University. Progress in Preventive Medicine. 5 (2).

REED, C. K. S. (2010) Intimate partner violence and infertility in Zambia (Doctoral dissertation).

SAMI, N. \& ALI, T. S. (2012) Domestic violence against infertile women in Karachi, Pakistan. Asian Review of Social Sciences. 1(1). P. 15.

SANCHEZ, N. (2014) A life course perspective on polycystic ovary syndrome. International Journal of Women's Health. 6. P. 115.

SHARMA, S. \& MISHRA, A. J. (2018) Tabooed disease in alienated bodies: A study of women suffering from Polycystic Ovary Syndrome (PCOS). Clinical Epidemiology and Global Health. 6 (3). Pp. 130-136.

SONI, N. R. (2017) Current Management on PCOS (Polycystic Ovary Syndrome)/ Stein-Leventhal Syndrome. Invest Gynecol Res Women's Health. 1 (3).

TABONG, P. T. N. \& ADONGO, P. B. (2013) Understanding the social meaning of infertility and childbearing: a qualitative study of the perception of childbearing and childlessness in Northern Ghana. PloS one. 8 (1).

TAN, S. et al. (2008) Psychological implications of infertility in women with polycystic ovary syndrome. Human Reproduction. 23 (9). Pp. 2064-2071. 
TAZUH, M. M. \& TOSAM, J. M. (2016) Being a woman in African culture: How culture shapes and defines the status of women. In: Tosam, J. M. \& Takov, P. (eds.) Philosophy in Culture: A Cross-Cultural Perspective. Mankon, Bamenda: Langaa RPCIG. Pp. 241-266.

TEEDE, H. J. et al. (2018) Recommendations from the international evidence-based guideline for the assessment and management of polycystic ovary syndrome. $\mathrm{Hu}$ man Reproduction. 33 (9). Pp. 1602-1618.

WHO (2017) [Online] Available from: https://www.who.int/news-room/fact-sheets/ detail/violence-against-women [Accessed: 9 September 2020].

WOLF, W. M. et al. (2018) Geographical prevalence of polycystic ovary syndrome as determined by region and race/ethnicity. International Journal of Environmental Research And Public Health. 15 (11). P. 2589.

WRIGHT, P. J., DAWSON, R. M. \& CORBETT, C. F. (2020) Social construction of biopsychosocial and medical experiences of women with polycystic ovary syndrome. Journal of Advanced Nursing. 76 (7). Pp. 1728-1736.

ZANGENEH, F. Z. et al. (2012) Psychological distress in women with polycystic ovary syndrome from Imam Khomeini Hospital, Tehran. Journal of Reproduction \& Infertility. 13 (2). P. 111. 